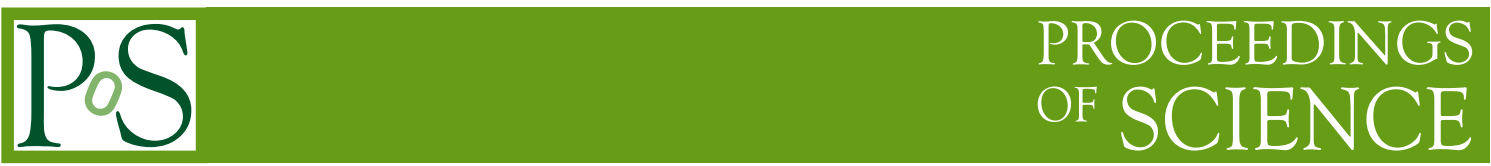

\title{
CP violation and cold electroweak baryogenesis in the Standard Model
}

\author{
Tomáš Brauner* \\ Faculty of Physics, University of Bielefeld, Germany \\ Department of Theoretical Physics, Nuclear Physics Institute ASCR, Řež, Czech Republic \\ E-mail: tbrauner@physik.uni-bielefeld.de
}

\begin{abstract}
Generating sufficient amount of $\mathrm{CP}$ violation that would account for the observed matterantimatter asymmetry in the universe is a long-standing problem in particle physics. It is a common lore that new physics beyond the Standard Model is needed for this purpose. This claim is based on earlier perturbative estimates of effective CP violation in the bosonic sector of the Standard Model induced by the quark CKM mixing as well as on the fact that the current lower limit on the Higgs boson mass rules out the possibility of a strongly first-order electroweak phase transition. We argue that within the cold electroweak baryogenesis scenario, there is still space for the baryon asymmetry to be generated solely from known electroweak physics. Sakharov's condition of being sufficiently far off equilibrium is satisfied by coupling the Higgs boson to a new field such as the inflaton. It is this coupling rather than a thermal electroweak transition what triggers the change of the sign of the Higgs boson mass squared in the fast expanding early universe. On the Standard Model side, we further develop the program initiated by Shaposhnikov, Smit, and others. We carry out a nonperturbative calculation of the lowest effective CP-violating operator in the bosonic sector at nonzero temperature. By inspecting the temperature dependence of the effective coupling, we conclude that cold electroweak baryogenesis is viable if the electroweak phase transition in the early universe occurs at the temperature below roughly $1 \mathrm{GeV}$.
\end{abstract}

XXIst International Europhysics Conference on High Energy Physics

21-27 July 2011

Grenoble, Rhône-Alpes France

\footnotetext{
* Speaker.
} 
Numerical simulations of the electroweak phase transition dynamics in the early universe are performed using an effective theory for the bosonic fields, obtained by integrating out the fermions. The latter amounts to the evaluation of the determinant of the Dirac operator for quarks or leptons in the background gauge and Higgs fields. This cannot be done analytically for arbitrary coordinatedependent background fields, and one therefore usually resorts to a covariant gradient expansion. Insofar CP violation in the leptonic sector of the Standard Model is neglected, which is a reasonable assumption compatible will all current experimental data, every CP-violating observable in the Standard Model must be proportional to the Jarlskog invariant, $J=\left|\operatorname{Im}\left(V_{i j} V_{k \ell} V_{i \ell}^{*} V_{k j}^{*}\right)\right| \approx 3 \times 10^{-5}$, constructed from the CKM matrix $V_{i j}$. Hence CP-violating effective bosonic operators can only appear from the fourth order of the gradient expansion on.

In fact, as shown by Smit [1], there appears to be no CP violation at the fourth order of the expansion. Two independent calculations of the sixth order at zero temperature were done [2, 3], giving nonzero, yet mutually contradicting results for the CP-violating effective action. Preliminary simulations [4] using the effective action of Ref. [2] yielded a result exceeding by roughly four orders of magnitude the observed value of the net baryon number density, normalized by the cosmic microwave background photon density, $n_{B} / n_{\gamma} \approx 6 \times 10^{-10}$. This is very encouraging for it demonstrates that the Standard Model can be capable of producing sufficient baryon asymmetry, in contrast to the common lore based on earlier perturbative estimates. We generalize here the calculation of Refs. [2,3] to nonzero temperature. Our motivation is to resolve the existing controversy, and to provide an upper bound on the phase transition temperature within the cold electroweak baryogenesis scenario.

The determinant of the Dirac operator in the covariant gradient expansion is evaluated using the method of covariant symbols (see Ref. [3] for details). The most naive generalization to nonzero temperature, based on a replacement of the loop frequency integral with a Matsubara sum, gives the following result for the CP-violating part of the Euclidean effective action for the Standard Model bosons, at the sixth order of the gradient expansion,

$$
\begin{aligned}
\Gamma_{\mathrm{eff}}= & -\frac{\mathrm{i}}{2} N_{c} J G_{\mathrm{F}} \kappa_{\mathrm{CP}} \int \mathrm{d}^{4} x\left(\frac{v}{\phi}\right)^{2}\left(\mathscr{O}_{0}+\mathscr{O}_{1}+\mathscr{O}_{2}\right), \\
\mathscr{O}_{0}= & -\frac{1}{3}\left(W^{+}\right)^{2} W_{\mu \mu}^{-} W_{v v}^{-}+\frac{5}{3}\left(W^{+}\right)^{2} W_{\mu \nu}^{-} W_{\mu v}^{-}-\frac{1}{3}\left(W^{+}\right)^{2} W_{\mu v}^{-} W_{v \mu}^{-}+\frac{4}{3} W_{\mu}^{+} W_{v}^{+} W_{\mu \alpha}^{-} W_{\alpha v}^{-}- \\
& -\frac{2}{3} W_{\mu}^{+} W_{v}^{+} W_{\mu \alpha}^{-} W_{v \alpha}^{-}-2 W_{\mu}^{+} W_{v}^{+} W_{\alpha \mu}^{-} W_{\alpha v}^{-}+\frac{4}{3} W_{\mu}^{+} W_{v}^{+} W_{\mu \nu}^{-} W_{\alpha \alpha}^{-}-\text {c.c. }, \\
\mathscr{O}_{1}= & \frac{8}{3}\left(Z_{\mu}+\varphi_{\mu}\right)\left[\left(W^{+}\right)^{2} W_{\mu}^{-} W_{v v}^{-}-\left(W^{+}\right)^{2} W_{v}^{-} W_{\mu v}^{-}-\left(W^{+}\right)^{2} W_{v}^{-} W_{v \mu}^{-}-\right. \\
& \left.-\left(W^{+} \cdot W^{-}\right) W_{\mu}^{+} W_{v v}^{-}+\left(W^{+} \cdot W^{-}\right) W_{v}^{+} W_{\mu \nu}^{-}+W_{\mu}^{+} W_{v}^{+} W_{\alpha}^{-} W_{\alpha v}^{-}\right]- \text {c.c. }, \\
\mathscr{O}_{2}= & 4\left(Z_{\mu} Z_{v}+\varphi_{\mu} \varphi_{v}\right)\left[\left(W^{+}\right)^{2} W_{\mu}^{-} W_{v}^{-}-\left(W^{-}\right)^{2} W_{\mu}^{+} W_{v}^{+}\right]- \\
& -\frac{16}{3}(Z \cdot \varphi)\left[\left(W^{+} \cdot W^{-}\right)^{2}-2\left(W^{+}\right)^{2}\left(W^{-}\right)^{2}\right]+\frac{4}{3}\left(Z_{\mu} \varphi_{v}+Z_{v} \varphi_{\mu}\right) \times \\
& \times\left[\left(W^{+}\right)^{2} W_{\mu}^{-} W_{v}^{-}+\left(W^{-}\right)^{2} W_{\mu}^{+} W_{v}^{+}-2\left(W^{+} \cdot W^{-}\right)\left(W_{\mu}^{+} W_{v}^{-}+W_{v}^{+} W_{\mu}^{-}\right)\right], \\
\kappa_{\mathrm{CP}}(T)= & \frac{\Delta}{G_{\mathrm{F}}} T \sum_{p_{0}=2 \pi n T} \int \frac{\mathrm{d}^{3} \boldsymbol{p}}{(2 \pi)^{3}}\left(p^{2}\right)^{3} \prod_{f=1}^{6} \frac{1}{\left(p^{2}+m_{f}^{2}\right)^{2}}, \quad \kappa_{\mathrm{CP}}(0) \approx 3 \times 10^{2} .
\end{aligned}
$$

Here $N_{c}=3$ is the number of colors, $G_{\mathrm{F}}$ the Fermi coupling constant, $\Delta$ the Jarlskog determinant, $\Delta=\left(m_{u}^{2}-m_{c}^{2}\right)\left(m_{c}^{2}-m_{t}^{2}\right)\left(m_{t}^{2}-m_{u}^{2}\right)\left(m_{d}^{2}-m_{s}^{2}\right)\left(m_{s}^{2}-m_{b}^{2}\right)\left(m_{b}^{2}-m_{d}^{2}\right)$, and $v \approx 246 \mathrm{GeV}$ the Higgs 


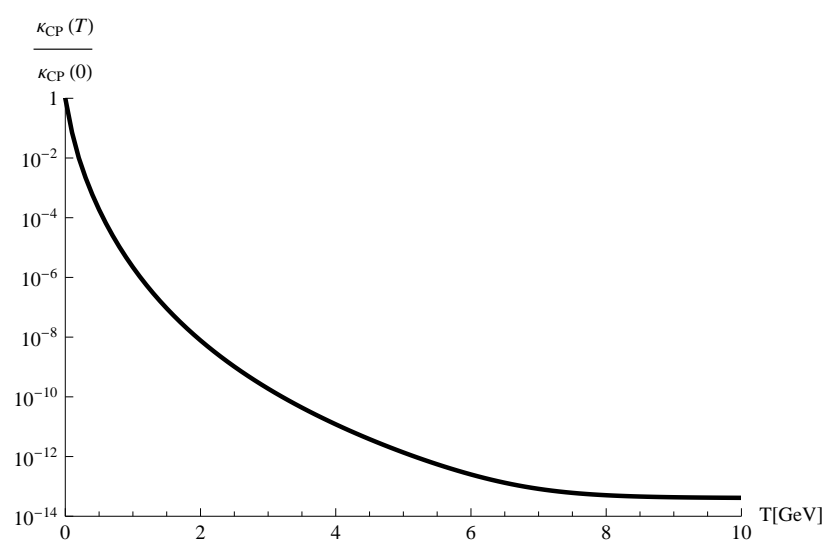

Figure 1: Effective coupling $\kappa_{\mathrm{CP}}$ as a function of temperature, normalized by its zero-temperature value.

expectation value. Also, $\varphi_{\mu}=\frac{1}{\phi} \partial_{\mu} \phi$ is the logarithmic derivative of the Higgs field $\phi$ and $W_{\mu v}^{ \pm}=$ $\partial_{\mu} W_{v}^{ \pm} \pm g^{\prime} B_{\mu} W_{v}^{ \pm}$the (hypercharge) covariant derivative of $W_{v}^{ \pm}$.

At zero temperature, our result exactly reproduces the finding of Ref. [3]. The temperature dependence of the effective coupling $\kappa_{\mathrm{CP}}$ is shown in Fig. 1. We can see that the coupling drops very steeply with temperature and at high temperatures, it reaches values compatible with the perturbative estimates, based on the dimensionless ratio $J \Delta / v^{12} \approx 10^{-24}$. The approximation scheme that we employed certainly has several caveats such as the use of the equilibrium thermal field theory in a far-off-equilibrium environment at the phase transition, the applicability of the gradient expansion for small values of the local Higgs field, and the neglect of strong-interaction effects at temperatures at or below $1 \mathrm{GeV}$, to mention some of them. Nevertheless, we believe to have provided motivation for a more detailed and systematic study of the cold electroweak baryogenesis scenario. More details on the computation will be provided in a forthcoming publication.

\section{Acknowledgments}

The presented results were obtained in collaboration with O. Taanila, A. Tranberg, and A. Vuorinen. This work was supported by the Sofja Kovalevskaja program of the Alexander von Humboldt Foundation.

\section{References}

[1] J. Smit, Effective CP violation in the standard model, JHEP 09 (2004) 067, [hep-ph/ 0407161$].$

[2] A. Hernandez, T. Konstandin, and M. G. Schmidt, Sizable CP Violation in the Bosonized Standard Model, Nucl. Phys. B812 (2009) 290, [arXiv:0810.4092].

[3] C. García-Recio and L. L. Salcedo, CP violation in the effective action of the Standard Model, JHEP 07 (2009) 015, [arXiv:0903.5494].

[4] A. Tranberg, A. Hernandez, T. Konstandin, and M. G. Schmidt, Cold electroweak baryogenesis with Standard Model CP violation, Phys. Lett. B690 (2010) 207, [arXiv: 0909 . 4199]. 